\title{
Prenatal Diagnosis and Counseling in Case of Congenital Hemivertebra
}

\author{
${ }^{1}$ Nupur Shah, ${ }^{2}$ Surinder K Gambhir
}

\begin{abstract}
Hemivertebra is a rare congenital disorder, where only one side of the vertebral body develops and it is even rarer to find it associated with a longitudinal bar. Prenatal ultrasound diagnostic features along with postnatal radiological correlation at 20 weeks gestation are presented in this report. Also, the relevant background, incidence, and associations are discussed for the clinicians especially obstetricians and radiologists to make them familiar with this condition.
\end{abstract}

Keywords: Congenital hemivertebra, Counseling, Prenatal diagnosis, Prenatal ultrasound.

How to cite this article: Shah N, Gambhir SK. Prenatal Diagnosis and Counseling in Case of Congenital Hemivertebra. Int J Infertil Fetal Med 2018;9(1\&2):19-21.

Source of support: Nil

Conflict of interest: None

\section{INTRODUCTION}

Hemivertebra is a rare congenital disorder, where only one side of the vertebral body develops, and it is even rarer to find it associated with a longitudinal bar., ${ }^{1,2}$ Other nomenclature used for this lesion are congenital scoliosis, unilateral aplasia of the vertebral body, and complete unilateral failure of formation of the vertebral body resulting in a laterally-based wedge vertebra with half a vertebral body, a single pedicle, and hemilamina. ${ }^{2,3}$ Prenatal ultrasound diagnostic features along with postnatal radiological correlation at 20 weeks gestation are presented in this report.

\section{CASE REPORT}

The patient was a 21-year-old primigravida, with no medical or obstetric risk factors. She has had no biochemical

\footnotetext{
${ }^{1}$ Consultant, ${ }^{2}$ Senior Consultant

${ }^{1}$ Department of Fetal Medicine, Institution Paras Bliss Hospital, Panchkula, Haryana, India

${ }^{2}$ Department of Obstetrics, Institution Paras Bliss Hospital, Panchkula, Haryana, India
}

Corresponding Author: Nupur Shah, Consultant fetal Medicine, Department of Fetal Medicine, Paras Bliss Hospital, Institution Paras Bliss Hospital, Panchkula, Haryana, India, e-mail: nupurmshah@gmail.com screening for aneuploidies and the NT scan done locally was normal (low risk). At the time of anomaly scan at 19 weeks, AC was below 5th centile suggestive of early fetal growth restriction (other parameters BPD, HC and FL were on 50th centile). The scan was performed by FMF (Fetal Medicine Foundation, UK) certified sonographer. The fetal spine showed a gross distortion to one side suggestive of fetal scoliosis. On closer examination, there was a triangular bony structure at the level of T10,11 (Fig. 1). The 3D reconstruction of the spine along with coronal view helped in diagnosing the unilateral fusion of the bodies of vertebrae raising the suspicion of longitudinal bar vertebrae. The skin over the spine was intact with no cranial signs excluding open spina bifida. There was no associated defect in the heart, GIT, limbs, kidneys (VACTREL). The final diagnosis was a hemivertebra. The couple declined the prenatal invasive testing by amniocentesis. They opted for termination of pregnancy which was done followed by radiological (Fig. 2) and gross examination (Fig. 3) of the stillborn fetus. These confirmed the prenatal ultrasound findings and the postnatal karyotype was normal (46XY).

\section{DISCUSSION}

Congenital hemivertebrae occurs in 0.5-1per 1000 live births and has a female preponderance (M:F ratio 0.31). ${ }^{1,2}$ In our case, it was a male, making this even rarer.

Embryologically, at the sixth week of gestation, two lateral chondrification centers arise and fuse at 8 weeks gestation to form primary ossification center of the vertebral body transiently separated anteriorly and posteriorly by notochord elements. So any defect in the above process leads to the hemivertebra (lateral or posterior), which acts as a triangular wedge-shaped defect causing contralateral spinal deviation. The ossification of the vertebral body progress in the following sequence: thoracic (8-12 weeks), lumbar (12 weeks), cervical and sacral (16-20 weeks onwards). ${ }^{4}$

Hemivertebra can be an isolated defect or associated with other defects (VACTERL-Vertebral, anal, cardiac, trachea esophageal, renal and limb abnormalities). It can be a part of rare genetic syndromes like Jarcho Levin, Klippel Fiel syndrome, etc. ${ }^{5}$

The prenatal diagnosis can be established as early as late first trimester with high-resolution machines and expertise, especially in lumbar and thoracic defects. However, 


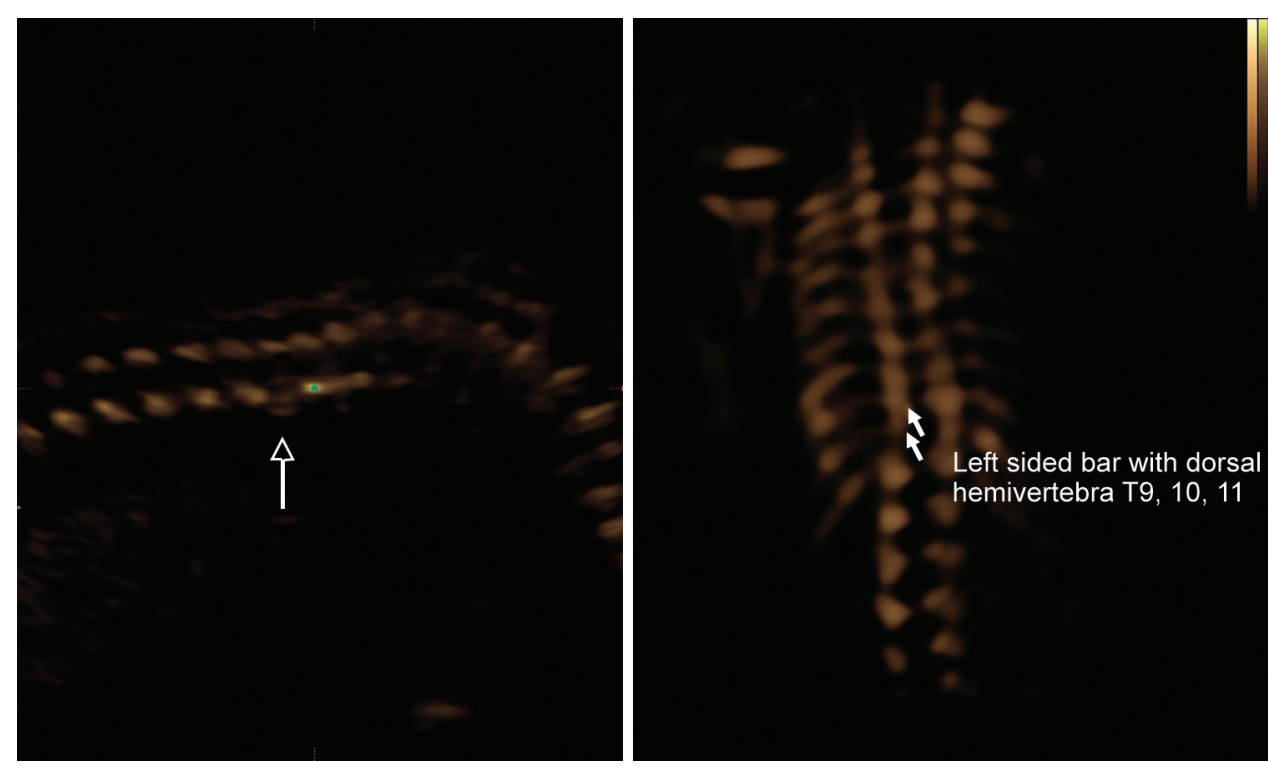

Fig. 1: Prenatal ultrasound in sagittal and coronal planes of the fetal spine shows a hemivertebra

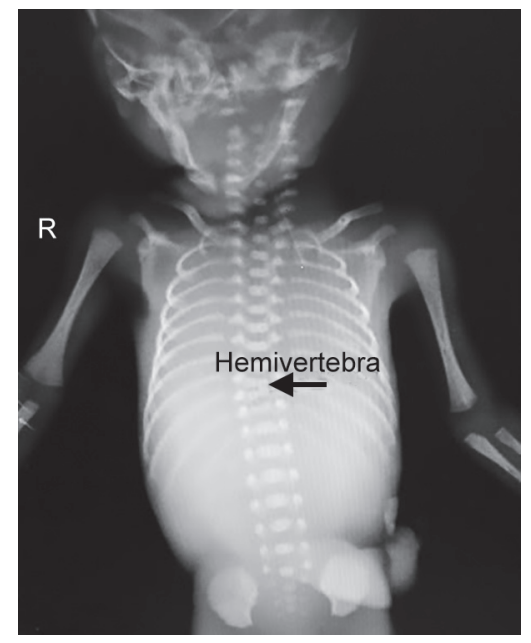

Fig. 2: Postabortal fetogram confirming the diagnosis and level of defect

hemivertebra is usually a second-trimester diagnosis. Once diagnosed, a thorough anatomical survey for associated structural defects should be made. Though chromosomal abnormalities are rare, a karyotype can be offered.

In isolated cases, the parents to be can be counseled to continue the pregnancy along with pediatric orthopedician consultation. Termination of pregnancy can be offered on parents' request and on the fact that all genetic syndromes cannot be diagnosed prenatally, but is not a rule of thumb in isolated cases due to the availability of postnatal orthopedic treatment. ${ }^{6}$ Postnatal management in isolated cases is aimed at achieving normal spinal curvature and the prognosis is usually good. Left untreated, $25 \%$ isolated cases will not progress, $50 \%$ will progress slowly whereas $25 \%$ will show rapid worsening. ${ }^{6}$ Prenatal diagnosis aids in rapid initiation of treatment and thus avoid worsening due to delayed treatment.

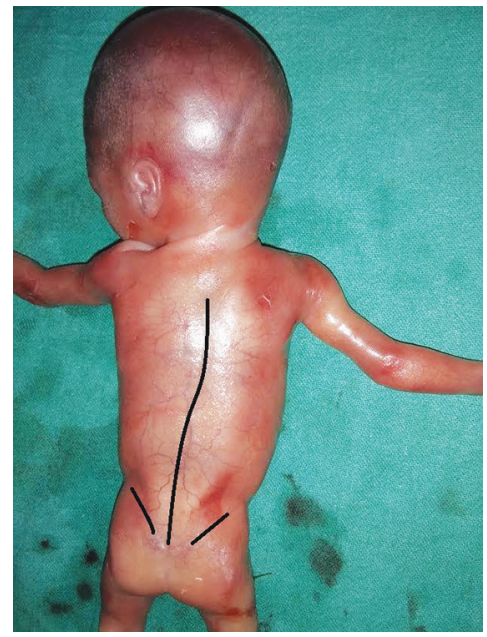

Fig. 3: Postabortal clinical picture showing gross scoliosis of the vertebral column and unequal level of iliac crests

In our case termination of pregnancy was due to parents' persistent request and unaffordability of specialized care and treatment. Termination of pregnancy is usually reserved in cases of associated structural or chromosomal/genetic abnormalities.

There is a very small risk of recurrence, and recurrence usually occurs in cases with associated anomalies. ${ }^{7,8}$

\section{CONCLUSION}

Congenital hemivertebra can be diagnosed prenatally and in isolated cases offers a valuable aid in the rapid initiation of the postnatal treatment for abnormal spinal curvature. This case report underscores the importance of the thorough anatomical survey and genetic counseling in fetal defects. It reiterates the fact that isolated cases of congenital hemivertebra do not necessitate termination of pregnancy and a prudent decision of the fate of preg- 
nancy should be made along with the parents' choice after thorough fetal structural and chromosomal evaluation.

\section{REFERENCES}

1. Weisz B, Achiron R, Schindler A, Eisenberg VH, Lipitz S, Zalel Y. Prenatal sonographic diagnosis of hemivertebra. J Ultrasound Med. 2004;23:853-857.

2. Varras M, Akrivis C. Prenatal diagnosis of fetal hemivertebra at 20 weeks gestation with literature review: International Journal of General Medicine 2010;3:197-201.

3. Gauthier DW, Meyer WJ. Hemivertebra. The-Fetus.net. Central nervous system. 1993;1-6. Available from: http:/ / www.thefetus. net. Accessed on May 14, 2010.
4. Nicolaides K, Sebire N, Snijders R, Ximenes R. The 11-14 weeks scan. www.sonoworld.com.

5. Wax JR, Watson WJ, Miller RC, Ingardia CJ, Pinette MG, Cartin A, et al. Prenatal sonographic diagnosis of hemivertebrae: associations and outcomes. Journal of Ultrasound in Medicine. 2008 Jul;27(7):1023-1027.

6. Winter RB. Congenital scoliosis. Orthop Clin North Am. 1988;19:395-408.

7. Textbook of Fetology 2nd Edition.Diana Bianchi, Fergal Malone, Mc Graw Hill 2010, Part 2-Section I, Chapter 88.

8. Goldstein I, Makhoul IR, Weissmaan A, Drugan A. Hemivertebra: Prenatal diagnosis, incidence and characteristics. Fetal Diagn Ther. 2005;20:121-126 